05.1;06.3;06.5;15

\title{
Локализация деформации в решетчатых структурах 3D-печатных образцов стали 03X17H14M2
}

\author{
(ㄱ Д.Г. Фирсов, С.Д. Конев, О.Н. Дубинин, С.А. Евлашин, И.В. Шишковский \\ Сколковский институт науки и технологий, Москва, Россия \\ "E-mail: i.shishkovsky@skoltech.ru
}

Поступило в Редакцию 6 апреля 2020г.

В окончательной редакции 6 апреля 2020г.

Принято к публикации 9 апреля 2020г.

\begin{abstract}
Методом цифровой корреляции изображения исследовано деформационное поведение в условиях сжатия созданных методом селективного лазерного плавления 3D-образцов стали 03Х17H14M2 с различными вариантами решетчатых структур. Изучены пространственно-временнб́е картины локализации поперечных $\left(\varepsilon_{x x}\right)$ и продольных $\left(\varepsilon_{y y}\right)$ деформаций актуальных типов топологических структур. Обнаружено, что возможно достижение относительной плотности $20 \%$ для $3 \mathrm{D}$-печатных изделий с решетчатыми $G$-структурами и размером ячейки 1.5 и $3 \mathrm{~mm}$. Однако при этом у всех решетчатых 3D-образцов исчезает пластичность, модуль Юнга уменьшается более чем на порядок, а коэффициент Пуассона возрастает в $1.3-2$ раза по сравнению с соответствующими параметрами для сплошных 3D-образцов.
\end{abstract}

Ключевые слова: метод корреляции цифровых изображений, селективное лазерное плавление, топологическая оптимизация, решетчатые структуры, локализация деформации.

DOI: 10.21883/PJTF.2020.14.49658.18329

Конструирование облегченных трехмерных (3D) изделий актуально во многих областях человеческой деятельности, где уменьшение массы основных силовых элементов означает увеличение полезной нагрузки (аэрокосмическая отрасль), экономию материалов на производстве и энергоэффективность (облегчение подвижных элементов различных установок), сближение с модулем упругости костной ткани, что является важной задачей биоматериаловедения $[1,2]$. Топологическая оптимизация (ТО) - один из самых быстрых и эффективных методов облегчения 3D-деталей, полученных методами аддитивных технологий, при сохранении их характеристик прочности и жесткости.

Целью ТО является определение оптимального распределения материала в области проектирования при заданных нагрузках с удовлетворением критериев и ограничений оптимизации [2,3]. В качестве критериев ограничения в рамках ТО в основном при использовании численных методов могут применяться различные функции (их комбинации), такие, например, как соответствие или потенциальная энергия деформации, объем, смещения или характеристики прочности. В настоящей работе для 3D-печатных структур и образцов с вариантами TO используется экспериментальный метод оптической диагностики, который называется методом цифровой корреляции изображений (DIC) $[4,5]$. Обсуждаются найденные параметры прочности и результаты оценки напряженнодеформированного состояния топологических решетчатых структур (ТРС).

В экспериментах использовался порошок нержавеющей стали 03Х17Н14M2 с размером частиц 20-53 $\mu \mathrm{m}$. 3D-печать образцов была выполнена методом селективного лазерного плавления (СЛП) [1]. Режим печати ре- комендован производителем установки: мощность лазера $113 \mathrm{~W}$, скорость сканирования лазера $700 \mathrm{~mm} / \mathrm{s}$, диаметр лазерного луча $55 \mu \mathrm{m}$, расстояние штриховки $50 \mu \mathrm{m}$, толщина одного слоя $20 \mu \mathrm{m}$. Стратегия сканирования в слое шахматная (квадраты со стороной $4 \mathrm{~mm}$ ).

Топологическое проектирование внутренней структуры трехмерных деталей (в настоящей работе в форме куба) было выполнено на основе возможностей встроенного программного обеспечения для подготовки к печати 3D-изделий, которое доступно на технологической установке TruPrint 1000. В нашем случае варианты структуры сетки моделировались с использованием программного обеспечения для 3D-печати Materialise Magics 22.0. На рис. 1, а показаны три характерных типа структур ( $G$-структуры, средний додекаэдр, ромбододекаэдр), которые были использованы в настоящей работе. Параметры элементарной ячейки и условия генерации сетки задавались нами уже в программном обеспечении nTopology Element (nTE, версия 1.24). Если выбран тип конструкции объекта (в нашем случае куб), nTE предлагает 22 варианта ячейки и 9 параметров заполнения. Мы задавали в nTE толщину ребер самой ячейки, которая по факту не может быть меньше диаметра лазерного луча. В перспективе нами планируется изучение влияния переменной толщины ребер ТРС, т.е. программное формирование градиента механических свойств изделия, путем создания „модификаторов“ (это могут быть точки, векторы или поверхности) и редактирования зависимости толщины элемента от расстояния до „модификатора“ путем редактирования свойств графики изделия в САПР (САПР - системы автоматизированного проектирования). С физической точки зрения изменение коэффициента заполнения ТРС (т. е. отношение объема, запол- 


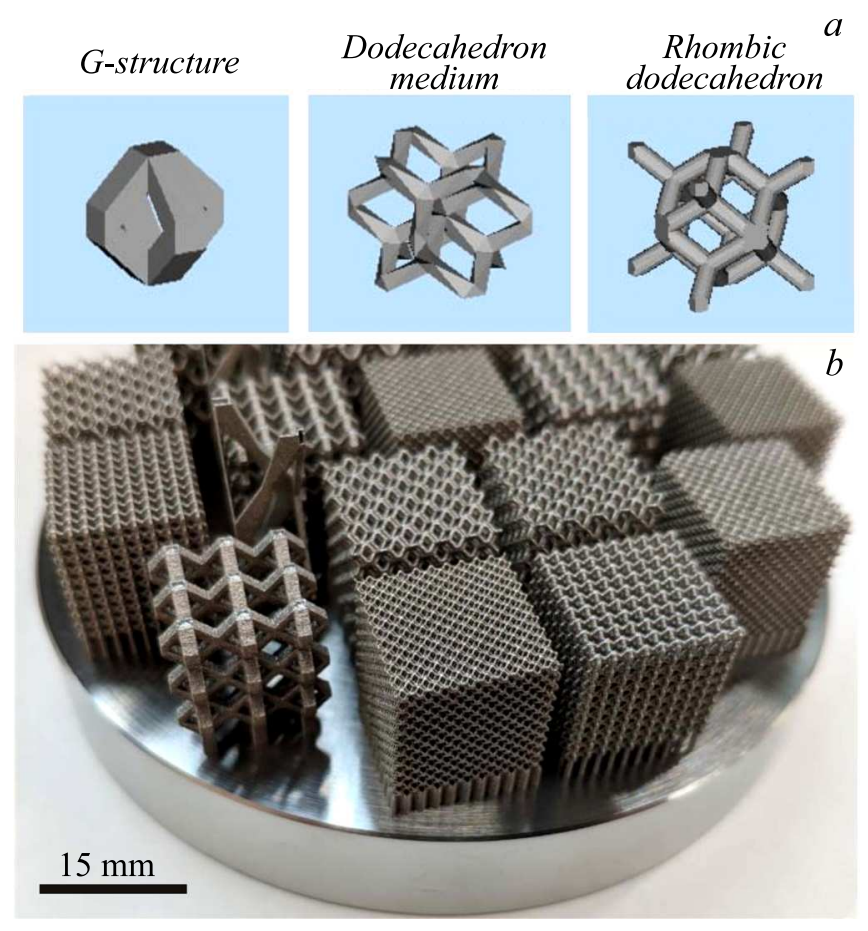

Рис. 1. $a-$ типы создаваемых структур, $b-$ внешний вид 3D-печатных TPC.

ненного материалом, к пустотам) влияет на плотность образца и его механические свойства. Проведенный нами топологический дизайн на стадии подготовки к 3D-печати показывает, что чем короче элементы, угол между осью которых с вертикалью составляет от 45 до $90^{\circ}$, тем больше вероятность того, что 3D-сетка будет успешно напечатана без деформации и подпорок.

Механическая нагрузка на сжатие проводилась на универсальной электромеханической испытательной системе Instron 5969 с постоянной скоростью сближения $2 \mathrm{~mm} / \mathrm{min}$ при комнатной температуре. Сравнивались механические характеристики топологических образцов (рис. $1, b)$ по измерениям в соответствии с ASTM E9.

Система бесконтактной трехмерной DIC использовалась во время механического испытания для получения трехмерных полей смещения на поверхности образца вплоть до разрушения. Специализированное программное обеспечение Vic-3D (Vic-Snap) от Correlated Solutions Inc. контролировало процесс съемки и обрабатывало полученные изображения с использованием двух цифровых 5MP камер Point Grey GRAS-50S5M-C. Математический аппарат программного обеспечения оптической системы основан на подходах DIC. Обе камеры были установлены на расстоянии $600 \mathrm{~mm}$ и под углом $30^{\circ}$ к образцу. Перед тестами проводилась калибровка системы 3D DIC. Ранее было показано [4], что такая видеосистема позволяет различать значения деформации с точностью, сравнимой с точностью установленного датчика динамической осевой деформации (согласно сертификату заводских испытаний). Чтобы понять природу изменения механических свойств образцов с вариацией ТРC, мы напечатали набор 3D-деталей для последующего механического испытания (рис. 1, $b$ ). Образцы представляли собой кубики с площадью поперечного сечения $15 \times 15 \mathrm{~mm}$, а размеры внутренних ячеек варьировались $(1.5,3$ и $5 \mathrm{~mm})$.

Испытание на сжатие позволило определить характеристики прочности, упругости и пластичности, такие как модуль Юнга, коэффициент Пуассона, предел текучести и характеристики деформационного упрочнения. Типичные инженерные кривые деформация-напряжение для 3D-печатных TPC сплава 03X17H14M2 представлены на рис. 2 (см. также таблицу). При аналогичном режиме 3D-печати сплошных образцов этого же сплава модуль Юнга был равен $75 \mathrm{GPa}$, предел прочности $575 \mathrm{MPa}$, коэффициент Пуассона 0.397, а удлинение достигало 70\%. Измеренная микротвердость сплошных 3D-печатных образцов из сплава 03Х17Н14M2 составила $190 \mathrm{HV}_{0.2}$. Как видно из рис. 2, если сравнивать с 3D-печатью сплошных образцов, практически все решетчатые образцы потеряли пластичность, модуль Юнга уменьшился на порядок и более, а коэффициент Пуассона возрос в $1.3-2$ раза. $G$-структуры с коэффициентом 1.5 и 3 имели максимальный модуль Юнга и коэффициент Пуассона. ТРС „средний додекаэдр“ показали минимальный модуль Юнга, а минимальный коэффициент Пуассона наблюдался для ТРС „ромбический додекаэдр“.

Сопоставление данных (см. рис. 2 и таблицу) показывает, что варьирование типа решетчатой структуры также существенно изменяет механические свойства образцов. Бо́льшую прочность имели образцы $G$-структуры (относительная плотность $\sim 22.42 \%$ от таковой для сплошного образца), далее следовал ромбический додекаэдр ( 19.32\%), и совсем низкую прочность имел средний додекаэдр ( 12.59\%). Это хорошо коррелирует с экспериментальными данными работы [6], в кото-

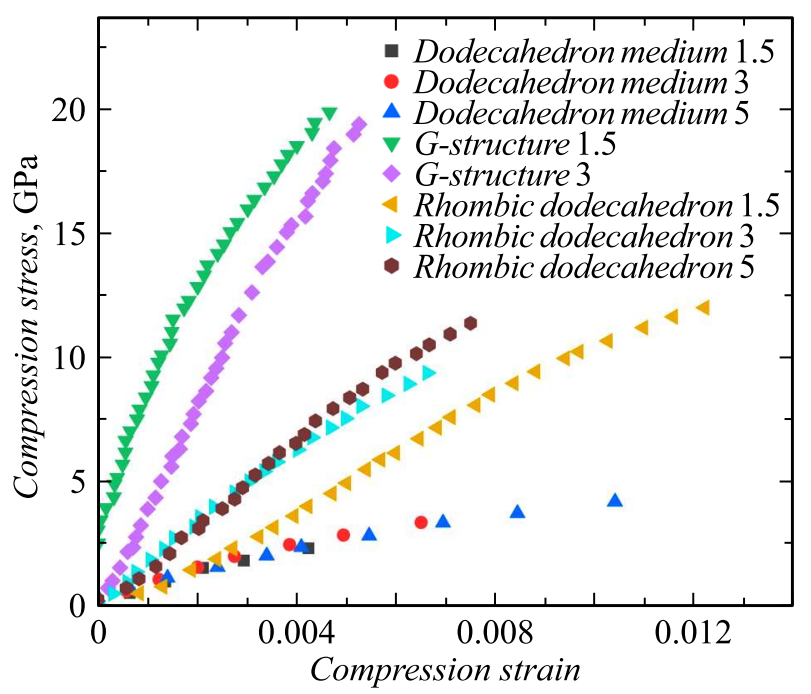

Рис. 2. Диаграмма деформация-напряжение для 3D-печатных топологических образцов. 
Механические свойства ТРС с вариацией размеров ячейки

\begin{tabular}{|c|c|c|c|c|c|c|c|}
\hline Тип структуры & $\begin{array}{c}\text { Коэффициент } \\
\text { заполнения } \\
\text { ячейки }\end{array}$ & $\begin{array}{c}\text { Пористость, } \\
\text { \% }\end{array}$ & $\begin{array}{c}\text { Модуль } \\
\text { Юнга, } \\
\text { GPa }\end{array}$ & $\begin{array}{c}\text { Коэффи- } \\
\text { циент } \\
\text { Пуассона }\end{array}$ & $\begin{array}{c}\varepsilon_{x x}, \% \\
(\max / \min )\end{array}$ & $\begin{array}{c}\varepsilon_{y y}, \% \\
(\max / \min )\end{array}$ & $\begin{array}{c}\varepsilon_{x y}, \% \\
(\max / \min )\end{array}$ \\
\hline$G$-структура & $\begin{array}{l}3 \\
1.5\end{array}$ & $\begin{array}{l}77.58 \\
77.58\end{array}$ & $\begin{array}{l}3.83 \\
3.69\end{array}$ & $\begin{array}{l}0.74 \\
0.80\end{array}$ & $\begin{array}{c}0.29 / 0.0 \\
0.096 /-0.001\end{array}$ & $\begin{array}{c}0.0 /-0.248 \\
0.0005 /-0.115\end{array}$ & $\begin{array}{c}0.119 /-0.033 \\
0.18 /-0.020\end{array}$ \\
\hline Ромбододекаэдр & 5 & 80.68 & 1.6 & 0.50 & - & - & - \\
\hline & 3 & 80.68 & 1.42 & 0.58 & $0.292 /-0.006$ & $0.0 /-0.278$ & $0.051 /-0.082$ \\
\hline & 1.5 & 80.68 & 1.08 & 0.50 & - & - & - \\
\hline Средний & 5 & 87.41 & 0.404 & 0.63 & - & - & - \\
\hline додекаэдр & $\begin{array}{l}3 \\
1.5\end{array}$ & $\begin{array}{l}87.41 \\
87.41\end{array}$ & $\begin{array}{l}0.49 \\
0.428\end{array}$ & $\begin{array}{l}0.66 \\
0.61\end{array}$ & $\begin{array}{c}0.314 /-0.002 \\
-\end{array}$ & $\begin{array}{c}0.002 /-0.266 \\
-\end{array}$ & $\begin{array}{c}0.0565 /-0.053 \\
-\end{array}$ \\
\hline
\end{tabular}

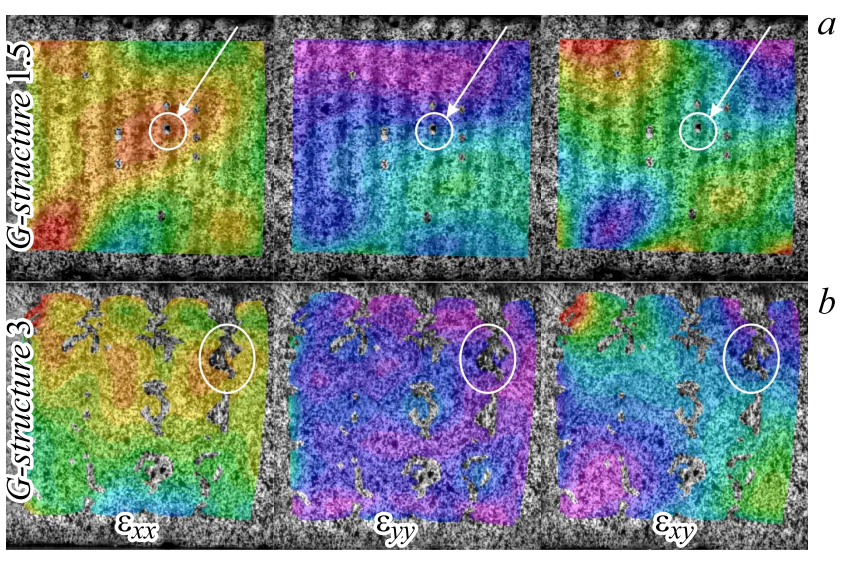

Рис. 3. $G$-структуры с размером ячейки $1.5(a)$ и $3 \mathrm{~mm}(b)$. Слева $-\varepsilon_{x x}$, в центре $-\varepsilon_{y y}$, справа $-\varepsilon_{x y}$.

рой было показано, что относительная плотность $20 \%$ при сравнении с плотностью сплошного 3D-материала оказалась соответствующей нижней границе для объемно центрированной кубической структуры с распорками по краям куба с размером ячеек $3 \mathrm{~mm}$. Таким образом, облегчение 3D-конструкции ячейками такого типа еще сохраняет стабильность изделия. С другой стороны, изменение коэффициента заполнения ячейки при фиксированном типе ТРС слабо влияет на прочностные параметры топологических 3D-образцов и не изменяет относительную плотность (см. таблицу, второй и третий столбцы). Причины такого поведения фактора заполнения ячейки в отношении механических свойств решетчатых структур нами пока не выяснены.

По результатам испытаний образцов на сжатие с использованием метода цифровой корреляции изображений построены поля поперечной, продольной и сдвиговой деформации на плоскости боковой грани $\left(\varepsilon_{x x}\right.$, $\left.\varepsilon_{y y}, \varepsilon_{x y}\right)$. Максимальные и минимальные значения компонентов тензора деформации приведены в таблице. На рис. 3, $a, b$ мы привели пример поперечных $\left(\varepsilon_{x x}\right)$, продольных $\left(\varepsilon_{y y}\right)$ и сдвиговых $\left(\varepsilon_{x y}\right)$ деформаций в конце сжатия образца для топологических конструкций с решетчатой $G$-структурой и размерами ячеек 1.5 и $3 \mathrm{~mm}$. Области черного цвета соответствуют местам зарождения разрушений образцов. Наиболее проблемные места выделены белыми кругами и овалами. Стрелками показано, как эти локализации выглядят при поперечных, продольных и сдвиговых нагрузках. Цвет сечения характеризует конфигурацию полей неоднородных деформаций. Наиболее загруженные области в электронной версии статьи окрашены в красный цвет (более светлые области в печатной версии), а наименее загруженные области - в синий цвет (более темные области). Полученные результаты фактически характеризуют прочность поверхностного сечения образца. Изменение степени наполнения (рис. 3, $a$ и $b$ ) показывает, что при меньшем размере ячейки $(1.5 \mathrm{~mm})$ максимальные значения поперечных и сдвиговых деформаций меньше (вторая строка таблицы). При размере ячейки $3 \mathrm{~mm}$ наблюдается большая неоднородность распределения поля напряжений.

Таким образом, в работе, насколько известно авторам, впервые получены и проанализированы следующие экспериментальные данные: локализация деформаций в решетчатых структурах 3D-печатных образцов стали 03X17H14M2; численные значения модуля Юнга, коэффициента Пуассона, предела текучести и характеристик деформационного упрочнения для трех типов часто используемых в топологическом дизайне решетчатых структур; влияние типа решетчатой структуры и размера

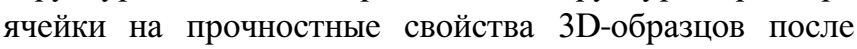
СЛП.

Цифровая оптическая система Vic-3D, которая была использована для анализа геометрии расположения концентраторов напряжений, позволяет достаточно точно локализовать место разрушения и увидеть динамику распределения нагрузки. Показано, что 3D-образцы с решетчатыми структурами по сравнению со сплошными теряют пластичность, их модуль Юнга уменьшается на порядок и более, а коэффициент Пуассона возрастает в 2 раза. Впервые обнаружено, что методом СЛП можно существенно снизить относительную плотность до $20 \%$ для 3D-печатных образцов с $G$-структурой и размерами 
ячейки 1.5 и $3 \mathrm{~mm}$, однако далее прочностные свойства изделий значительно ухудшаются.

\section{Благодарности}

Авторы благодарны nTopology Element Inc. за возможность использования программного обеспечения.

\section{Финансирование работы}

Работа частично финансировалась в рамках проекта Единого отраслевого тематического плана ЕОТП-МТ-097 Госкорпорации Росатом.

\section{Конфликт интересов}

Авторы заявляют, что у них нет конфликта интересов.

\section{Список литературы}

[1] Шишковский И.В. Основы аддитивных технологий высокого разрешения. СПб.: Питер, 2016. 400 с.

[2] Wang X., Xu S., Zhou S., Xu W., Leary M., Choong P., Choong P., Qian M., Brandt M., Xie Y. // Biomaterials. 2016. V. 83. P. 127-141. DOI: 10.1016/j.biomaterials.2016.01.012

[3] Liu J., Gaynor A.T., Chen S., Kang Z., Suresh K., Takezawa A., Li L., Kato J., Tang J., Wang C., Cheng L., Liang X., To A. // Struct. Multidiscip. Optim. 2018. V. 57. P. 2457-2483. DOI: $10.1007 / \mathrm{s} 00158-018-1994-3$

[4] Sutton M.A., Orteu J.J., Schreier H. Image correlation for shape, motion and deformation measurements. Basic concepts, theory and applications. Springer, 2009. $364 \mathrm{p}$.

[5] Gamboa C.B., Martin-Béjar S., Vilches F., López G., Hurtado L. // Materials. 2019. V. 12. P. 4156-4170. DOI: $10.3390 / \mathrm{ma} 12244156$

[6] Sola A., Defanti S., Mantovani S., Merulla A., Denti L. // 3D Printing Additive Manufactur. 2020. V. 7. N 1.

DOI: $10.1089 / 3 \mathrm{dp} .2019 .0119$ 